Revista Complutense de Educación

ISSNe: 1988-2793

https://dx.doi.org/10.5209/rced.63393

\title{
Aprendizaje-Servicio. Los retos de la evaluación
}

Autores: Marta Ruiz-Corbella y Juan García-Gutiérrez (edits.)

Editorial: Narcea

Año de publicación: 2019

$N^{o}$ de páginas: 206

ISBN: 978-84-277-2531-7

Los autores de esta obra, Marta Ruiz Corbella y Juan García Gutiérrez, editan un trabajo muy detallado sobre los retos de la evaluación en el Aprendizaje-Servicio. Publicada en 2019, cuenta con la participación de numerosos autores especialistas en metodología Aprendizaje-Servicio. Tratan una de las vertientes que requiere de un mayor estudio en esta metodología: la evaluación. Es indudable, tal y como indican los editores en la introducción del libro, que la universidad está en una profunda transformación, especialmente en la actividad docente. Y este cambio está viniendo de la mano de diversas metodologías innovadoras, entre las que se encuentra el Aprendizaje-Servicio. Sin embargo, un aspecto aún pendiente es la evaluación de este tipo de proyectos. Para ello, en esta obra se agrupan en once capítulos los criterios básicos que debe atender el proceso de evaluación de aprendizajes y proyectos de ApS.

El primer capítulo se plantea la siguiente cuestión en su título: ¿Es posible evaluar los resultados de proyectos de Aprendizaje-Servicio? Se incide en la importancia de la evaluación para el logro de evidencias de éxito y, para ello, se realiza un recorrido por los principales indicadores que nos permiten evaluar el impacto del ApS en la universidad. La lectura de este capítulo permite un cuidadoso acercamiento sobre la importancia que tiene la correcta evaluación de los proyectos de ApS.

A continuación, se abordan los diferentes elementos clave que se deben tener en cuenta en los procesos de evaluación de proyectos de Aprendizaje-Servicio, ya que en muchas ocasiones se recogen datos para una evaluación posterior, pero sin tener claro el objetivo de evaluación y, por lo tanto, de la recolección de datos. Se detalla, además, el papel de cada uno de los participantes en el proceso evaluativo. Finalmente, los autores plantean los retos de la evaluación de una forma muy sintética y acertada.

El capítulo tercero se centra en la evaluación como una medición del impacto de la innovación educativa. Para dicha medición, se recoge una explicación detallada de los casos prácticos de la Universidad Politécnica de Madrid y la Universidad Pompeu Fabra. Ambas universidades realizan la evaluación del ApS a través de proyectos de innovación y realizan una evaluación del impacto que estos proyectos ha tenido en la innovación docente.

El cuarto capítulo nos lleva a una cuestión muy relevante si estamos pensando en llevar a cabo una experiencia de ApS y es la evaluación durante el diseño y planifi- 
cación del programa, es decir, evaluación ex-ante. Este tipo de evaluación nos indica que son necesarias una serie de características en las diferentes fases y componentes del diseño y planificación de un programa de intervención.

Continuamos la lectura de la obra con un capítulo destinado a los criterios de calidad para evaluar proyectos de $\mathrm{ApS}$, basados en un proyecto de investigación que buscaba validar un modelo de institucionalización del ApS en la universidad española. Las autoras concluyen con dos elementos que son básicos, bajo su experiencia en el proyecto de investigación, para lograr institucionalizar de manera formal los proyectos de Aprendizaje-Servicio en la universidad: La formación de los docentes y una cultura de evaluación. Para su consecución se debe seguir trabajando en la consolidación de los diseños y métodos de evaluación de los proyectos, así como el uso de investigaciones longitudinales para conocer la eficacia de éstos.

En el siguiente capítulo, se afirma que los proyectos de Aprendizaje-Servicio son el escenario perfecto para desarrollar sistemas de evaluación que se basen en el diálogo y reflexión, como, por ejemplo, la evaluación participativa. Para ello se desarrolla una aproximación de dicho modelo que nos permite ver el aspecto en común entre este tipo de evaluación y los proyectos ApS: la participación. Además, dentro del marco de la evaluación participativa, los autores afirman que se debe tener presente la participación de la institución comunitaria en la evaluación para lograr así un adecuado desarrollo de los proyectos de Aprendizaje-Servicio.

En el séptimo capítulo, nos encontramos con un estado de la cuestión sobre la evaluación del aprendizaje de competencias curriculares en los proyectos de Aprendizaje-Servicio. Las autoras han detectado la carencia de bibliografía sobre proyectos de ApS que evalúe el impacto académico en los estudiantes, eje fundamental en un proyecto de este tipo. Por ello, plantean la importancia de realizar una evaluación de competencias de una manera íntegra y finalizan el capítulo con una presentación muy acertada de las herramientas de evaluación de competencias que la literatura académica aporta.

En el siguiente capítulo nos encontramos un abordaje más reflexivo y global del proceso de evaluación enmarcado en el desarrollo de la competencia ética y compromiso cívico en las Universidades actuales. Los autores realizan una clarísima reflexión sobre los aspectos que se asocian al desarrollo de la "competencia ética" y el "compromiso cívico" en las universidades. En el análisis de estos aspectos, se detecta una dificultad para lograr evaluar aprendizajes éticos o cívicos, lo que lleva a los autores a realizar una propuesta de evaluación de este tipo de competencias entre los estudiantes, como sería el uso de rúbricas y cuadernos de campo. Ambas herramientas se desarrollarán en los capítulos siguientes.

En el capítulo nueve, nos encontramos una propuesta para el uso de rúbricas en la evaluación de proyectos de Aprendizaje-Servicio, articulada desde dos objetivos claros: el valor de la evaluación del ApS de forma colaborativa e integral y la rúbrica como herramienta que pone en práctica este tipo de evaluación. Para abordar ambos objetivos se realiza un recorrido deductivo desde un marco general de la evaluación hasta el detalle preciso de la herramienta de rúbrica para la evaluación de proyectos.

Al igual que el título anterior, el siguiente capítulo elabora una propuesta de evaluación de ApS a través del diario de campo, el cual se puso en práctica en dos proyectos que se detallan de una manera muy clarificadora a lo largo del capítulo. Este tipo de herramienta es para los autores útil en contextos muy diferentes, ya 
que permite adaptar su uso y estructura a la necesidad del entorno en que se dan los proyectos de Aprendizaje-Servicio.

En el último capítulo de esta obra, nos encontramos con un cierre muy acertado a la temática tratada en toda la obra: la evaluación, pero en este caso, de los procesos de institucionalización del ApS, tanto en escuelas como universidades. Para ello se realiza un abordaje del concepto de institucionalización del Aprendizaje-Servicio, junto a un modelo de evaluación destinado a tal cuestión. Por último, los autores realizan un análisis de los indicadores a instrumentos relacionados con la evaluación institucional.

La obra concluye con un desarrollado espacio de bibliografía que aporta una mayor profundización a la evaluación de los proyectos de Aprendizaje-Servicio. Nos encontramos ante un libro muy destacable por el extenso análisis que realizan los autores de un tema que requiere de un abordaje por parte de los profesionales implicados en la puesta en marcha de proyectos de Aprendizaje-Servicio. Es un documento de gran utilidad tanto para los expertos que ya están poniendo en práctica esta metodología en sus aulas, como para los profesionales que buscan iniciarse en el Aprendizaje-Servicio con una perspectiva evaluativa desde su comienzo.

María Aránzazu Carrasco Temiño mcarrasco@ucm.es Universidad Complutense de Madrid 